\section{Bleaching melanin in formalin- fixed and paraffin-embedded melanoma specimens using visible light: A pilot study}

\author{
Claudio Pigoli, ${ }^{1}$ Lucia Rita Gibelli, ${ }^{2}$ \\ Mario Caniatti, ${ }^{1}$ Luca Moretti, ${ }^{3}$ \\ Giuseppe Sironi, ${ }^{1}$ Chiara Giudice ${ }^{1}$
}

${ }^{1}$ Department of Veterinary Medicine (DIMEVET), University of Milan, Lodi ${ }_{2}^{2}$ Istituto Zooprofilattico Sperimentale della Lombardia e dell'Emilia Romagna, Milan

${ }^{3}$ Department of Physics, Politecnico di Milano, Milan, Italy

\begin{abstract}
In fluorescence microscopy, light radiation can be used to bleach fluorescent molecules in formalin-fixed and paraffin-embedded (FFPE) samples, in order to increase the ratio between signal of interest and background autofluorescence. We tested if the same principle can be exploited in bright field microscopy to bleach pigmented melanoma FFPE sections together with cell morphology maintenance. After dewaxing and rehydration, serial FFPE sections of a feline diffuse iris melanoma, a canine dermal melanoma, a gray horse dermal melanoma and a swine cutaneous melanoma were irradiated with visible light for 1, 2, 3, 4 and 5 days, prior to Hematoxylin \& Eosin staining. Complete bleaching was obtained after 1-day treatment in feline and swine melanomas, while 2 and 3 days were required in canine and equine neoplasms, respectively. In all treated samples, cell morphology was maintained. Photo-induced bleaching combined with immunohistochemistry was tested after a 3-day photo-treatment using five different markers. According to the literature, in all samples neoplastic cells stained positive for vimentin, S100 and PNL2, while negative for FVIII and pancytokeratin. In conclusion, visible light can be effectively exploited to bleach pigmented melanoma FFPE sections prior to perform routine histochemical and immunohistochemical stains.
\end{abstract}

\section{Introduction}

Melanins is a generic term derived from the Greek word "melanos" meaning dark. Although the term melanin is often used to identify a single entity, it actually encompasses a number of pigments widely distributed in living beings and characterized by different and partly unknown molecular features. In general, melanins can be defined as a heterogeneous group of molecules deriving from the oxidation of phenolic compounds with polymerization of the resulting chemical products. In animals, melanins are subdivided into eumelanins, black to brown in color, and pheomelanins, reddish to yellowish. ${ }^{1}$ A third subtype of melanin, called neuromelanin and often associated to the afore-mentioned groups, is composed of a mixture of eumelanins and pheomelanins. In particular, it has been hypothesized that neuromelanin granules consist of a pheomelanin core surrounded by a eumelanin layer. ${ }^{2}$

In animals, melanin granules are present in various sites including integumentary system, eye, central nervous system and inner ear. Given its presence in a wide range of tissues, it is not surprising that melanin performs different tasks ranging from merely mechanical ones to those of radical scavengers. The main role of melanin is to protect living organisms from the harmful effects of electromagnetic radiations. In particular, it is able to absorb radiations in a wide range of wavelengths from the ultraviolet to the visible spectrum. The energy absorbed is then redistributed as heat. ${ }^{1}$ Energy absorption is also followed by redox reactions that ultimately can lead to formation of molecules having spectral absorption properties that differ from the original polymer. Since melanins absorb both visible and not visible light radiation, their oxidation can be achieved both by high-energy radiation, such as ultraviolet rays, and by low-energy radiation, such as visible light. The phenomenon by which a molecule undergoes oxidation by means of light irradiation is called photo-oxidation. ${ }^{3}$ Although the exact mechanism of melanin photo-oxidation has not yet been explained in all its aspects, several studies have shown that during ultraviolet and visible light irradiation, singlet oxygen and superoxide anions are produced and rapidly quenched by melanin. The oxidation induced by these reactive oxygen species is considered to play an important role in the photo-degradation of melanin itself. ${ }^{3,4}$ Visible light is routinely used, in our laboratory, to damage the molecules responsible for background autofluorescence, thus making them no longer fluorescent, prior to perform immunofluorescence assays on formalin-fixed and paraffin-embedded (FFPE) samples. ${ }^{5-8}$ In the present study, we investigated if the same principle could also be exploited in bright field microscopy to bleach FFPE samples of pigmented melanoma, prior to perform routine histochemical and immunohistochemical stains, ensuring the maintenance of cell morphology.
Correspondence: Claudio Pigoli, Department of Veterinary Medicine (DIMEVET), University of Milan, Via dell'Università 6, 26900 Lodi, Italy.

Tel. +39.02.50334165.

E-mail: claudio.pigoli@unimi.it

Key words: Bright-field microscopy; canine; equine; feline; light radiation; photobleaching; swine.

Contributions: CP, LRG, MC, LM, GS, CG, study design, data analysis and interpretation; LM, performing of the light source spectral measurement; $\mathrm{CP}$, performing of the photobleaching protocol, histochemical and immunohistochemical stains; CP, LRG, MC, GS, CG, viewed the histological specimens. All the authors have drafted the manuscript and reviewed the work critically, have read and approved the final manuscript and agreed to be accountable for all aspects of the work.

Conflict of interest: the authors declare they have no competing interests.

Acknowledgements: We would like to thank Massimo Datteri and Laura Tassielli from the Istituto Zooprofilattico Sperimentale della Lombardia e dell'Emilia Romagna for the technical support, cutting and staining of histological sections. We would also like to thank Stefano Tomasoni from DI.C.S.EL srl for his valuable advices regarding the world of LED light sources.

Received for publication: 9 September 2019 Accepted for publication: 15 October 2019.

This work is licensed under a Creative Commons Attribution-NonCommercial 4.0 International License (CC BY-NC 4.0).

CCopyright: the Author(s), 2019

Licensee PAGEPress, Italy

European Journal of Histochemistry 2019; 63:3071 doi:10.4081/ejh.2019.3071

\section{Materials and Methods}

\section{Samples}

In this study, four FFPE samples of pigmented melanocytic neoplasms were used. In particular, a grey horse dermal melanoma and a swine cutaneous melanoma were retrieved from the archives of the Istituto Zooprofilattico Sperimentale della Lombardia e dell'Emilia Romagna (IZSLER), while a case of feline diffuse iris melanoma and a case of canine dermal melanoma were obtained from the archives of the Department of Veterinary Medicine, University of Milan. For each sample, six 4 $\mu \mathrm{m}$-thick serial sections were cut and placed on SuperFrost Plus ${ }^{\mathrm{TM}}$ adhesion slides (Thermo Scientific, Waltham, MA, USA). 
Light source and emission spectrum evaluation

In this study a $300 \mathrm{~W}$ Viugreum ${ }^{\mathrm{TM}}$ spotlight (Guangdong Zhongshan Yilangde Illumination CO. Ltd., Zhongshan City, China) containing a 594-phosphor white LED panel with an emission divergence of $120^{\circ}$ and emitting a 36000 lum and $6500 \mathrm{~K}$ light, was used. Since the manufacturer of the spotlight supplies only the color temperature of the light emitted but not the specific emission spectrum, the latter was measured using a HR2000+ spectrometer (Ocean Optics, Largo, Florida), which has a wavelength range of detection from $190 \mathrm{~nm}$ to $1100 \mathrm{~nm}$. The light of the lamp has been coupled to the spectrometer through an optical fiber.

\section{Photobleaching apparatus}

The photobleaching apparatus has been made similarly to the one described by Duong and Ha. ${ }^{6}$ The spotlight was placed in a room maintained at $4^{\circ} \mathrm{C}$ and placed so that the LED panel was facing upwards and that $4.5 \mathrm{~cm}$ thick reflective spacers could be placed on the spotlight protective glass. With this configuration the distance between the samples and the LED panel was $8.8 \mathrm{~cm}$. Above the spacers, two $12-\mathrm{cm}$ square Petri dishes were placed, filled with TRIS buffer $\mathrm{pH} 7.6$ added with $0.05 \%$ sodium azide, in order to inhibit bacterial growth (Figure 1). A glass mirror was positioned on the Petri dishes so that the light not directly absorbed by the samples could, at least in part, be redirected towards them. With this configuration of the apparatus, the amount of light impinging directly on the samples from the LEDs has been calculated to be around $0.455 \mathrm{~mW} / \mathrm{cm}^{2}$ (derived from the LED panel parameters declared by the manufacturer and measured spectrum).

\section{Temperatures reached by the sections during photobleaching treatment}

To evaluate the temperature reached by the sections during treatment, during a preliminary experiment, an alcohol thermometer was placed in the TRIS buffer-filled Petri dishes of the photobleaching apparatus. The temperatures were recorded before starting the treatment and, once started, at 1 -h intervals for $12 \mathrm{~h}$.

\section{Photobleaching protocol combined with routine histochemical stain}

Sections were dewaxed in xylene, rehydrated through graded alcohols and then placed in $0.05 \%$ sodium azide TRIS buffer. The sections were inserted into the Petri dishes and the photobleaching protocol was started. A control section for each sample was dipped in $0.05 \%$ sodium azide TRIS buffer maintained at $24^{\circ} \mathrm{C}$ (the temperature reached by the sections during photo-treatment) and in the dark inside a thermostat incubator (Cavallo srl, Buccinasco, MI, Italy). Every $24 \mathrm{~h}$, for 5 days, a section for each sample was removed from the Petri dishes and stored with the control sections. At the end of the fifth day, to minimize the possible differences given by a non-contemporary histochemical staining, all histological sections were jointly stained with hematoxylin-eosin (H\&E).

\section{Photobleaching protocol combined with immunohistochemical stain}

In the immunohistochemical assay, five different markers were tested: three of them (vimentin, S100 and PNL2) are commonly used in the melanocytic neoplasm histological identification workflow, while two (pancytokeratin and FVIII) are usually not expressed by melanoma cells. Sections were dewaxed in xylene and rehydrated through graded alcohols; endogenous peroxidase activity was blocked with $0.3 \%$ $\mathrm{H}_{2} \mathrm{O}_{2}$ in methanol for $30 \mathrm{~min}$. Sections were then dipped in Petri dishes containing $0.05 \%$ sodium azide TRIS buffer and photobleached for 3 days. The duration of the photo-treatment was chosen after the evaluation of the results of photobleaching and H\&E combined protocol: the treatment able

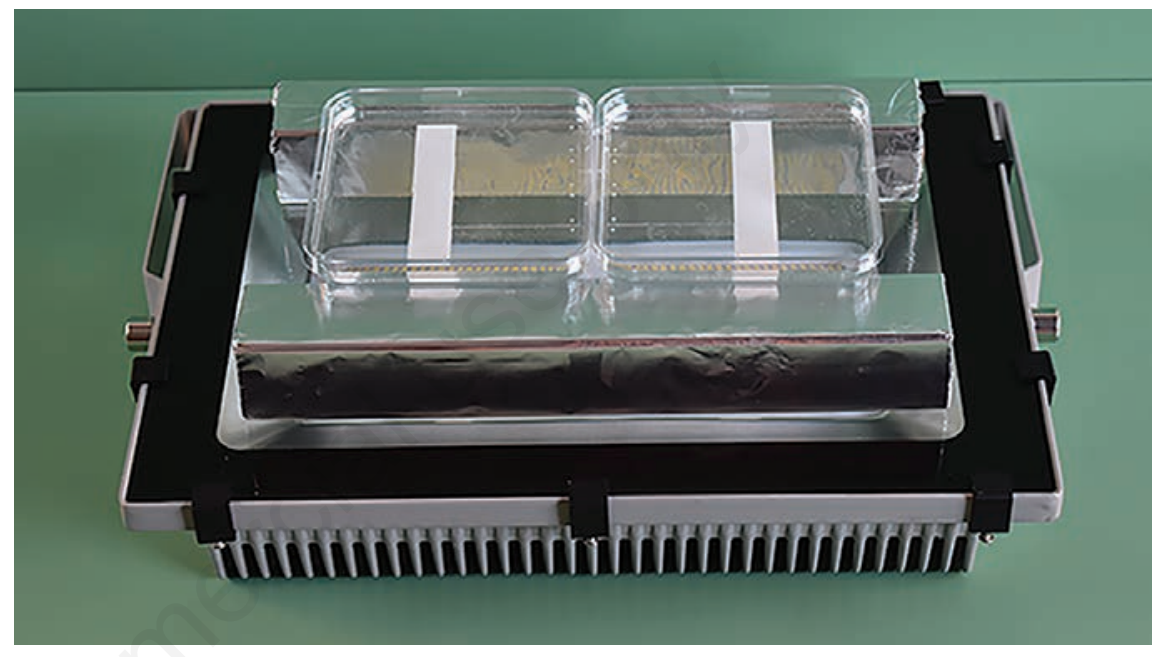

Figure 1. Photobleaching apparatus. The spotlight was placed so that the LED panel was facing upwards and that reflective spacers could be placed on the spotlight protective glass. Above the spacers, two $12-\mathrm{cm}$ square Petri dishes, filled with TRIS buffer added with $0.05 \%$ sodium azide, were placed. In the image, the covering glass mirror is not visible.

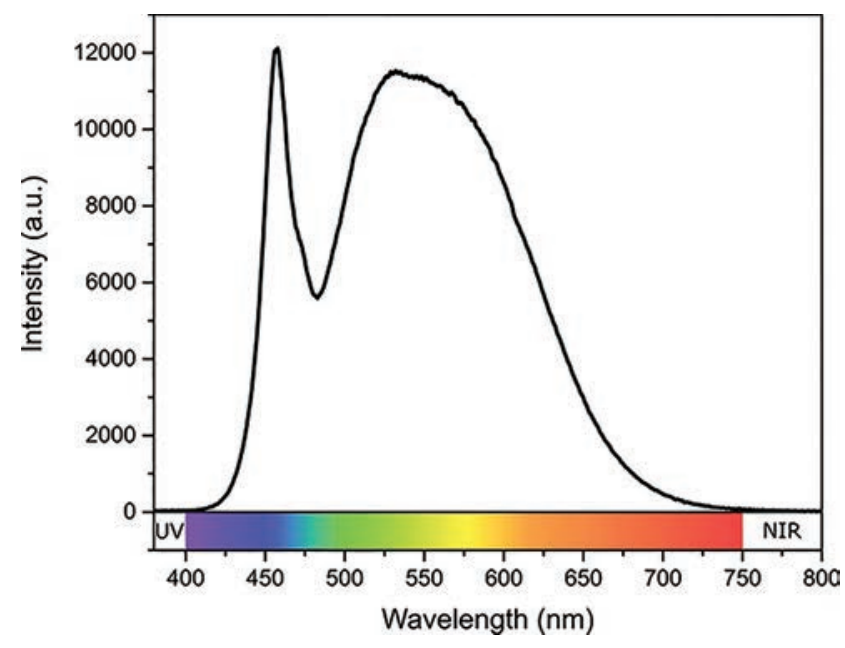

Figure 2. Light source emission spectrum. Emitted radiation is in the visible range $(-400-$ $750 \mathrm{~nm}$ ), showing main peaks in the blue and green-yellow ranges. The colored stripe in the bottom of the graph roughly represent how different wavelengths would be perceived by human eye. 

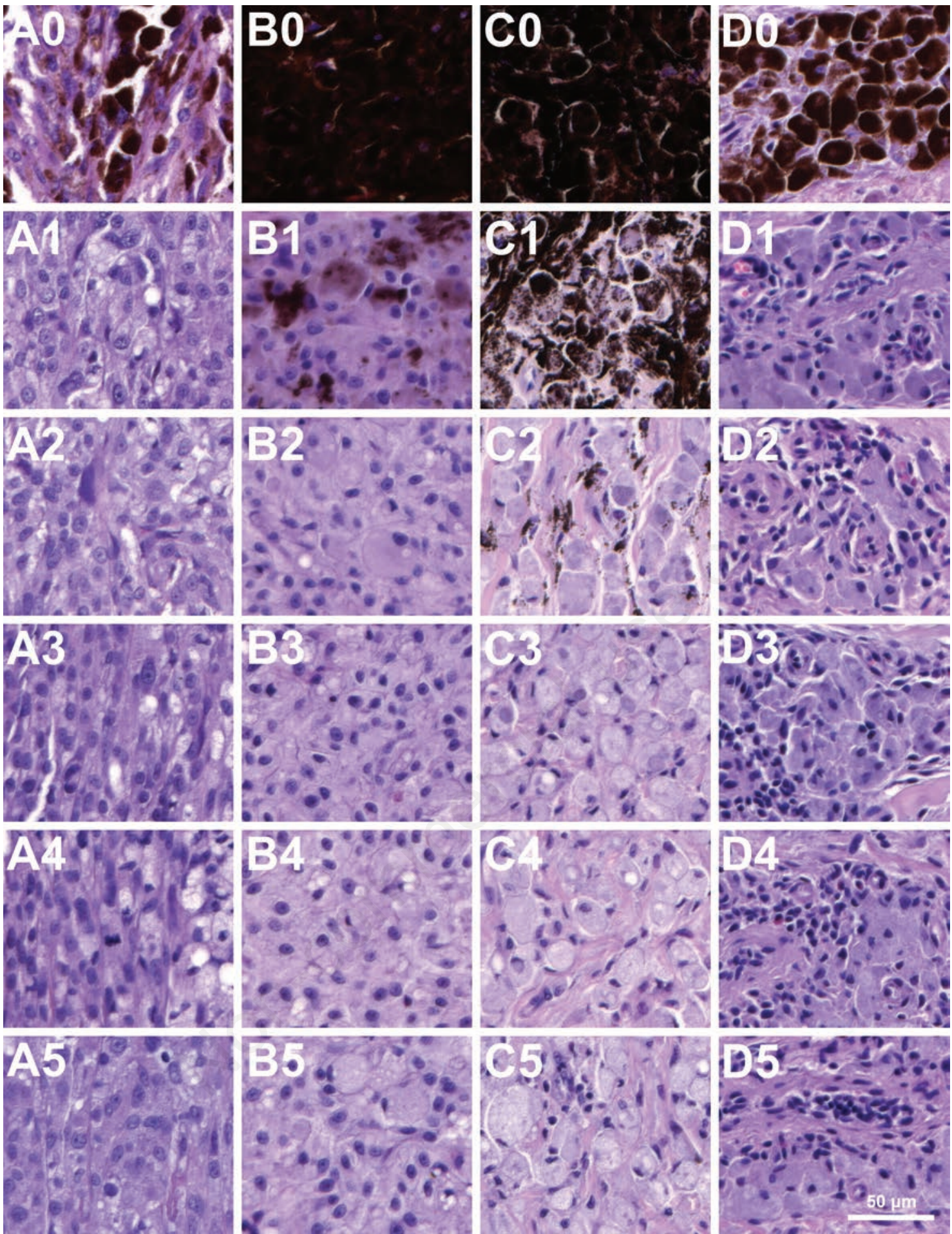

Figure 3. Effect of the photo-treatment on feline diffuse iris melanoma (A), canine dermal melanoma (B), gray horse dermal melanoma (C) and swine cutaneous melanoma (D) FFPE samples. In the figure untreated (0) and 1-day (1), 2-day (2), 3-day (3), 4-day (4), 5-day (5) treated sections are shown. A0-5) In the feline sample, after a 24-h photobleaching treatment, neoplastic cells were completely depigmented and no differences were noted for longer treatments; in all treated sections, cell morphology was well maintained. B0-5) In the canine sample, a 24-h treatment resulted in a considerable melanin degradation; still, single or aggregated neoplastic melanocytes, either totally black or characterized by a black-brownish cytoplasm, were observed; following a 2-days treatment, neoplastic cells were entirely depigmented and no further changes were noted in the 3-, 4- and 5-days treatments. In all treated sections, cell morphology was well maintained. $\mathrm{C0}$-5) In the equine sample after a 24-h treatment, a considerable part of the section was still pigmented, with melanocytes characterized by a totally black cytoplasm or variable numbers of intracytoplasmic round black granules; after a 2-days treatment, occasional melanocytes with intracytoplasmic granules were still present; melanin was not observed in the 3-, 4- and 5-days treated sections. In all treated sections, cell morphology was well maintained. D0-5) In the swine sample, pigmented cells were completely bleached after a 24-h treatment; no changes were observed following longer treatments; in all treated sections, cell morphology was well maintained. H\&E staining. 
to ensure the complete depigmentation of all samples was applied. Following photobleaching, different antigen retrieval methods were applied: pepsin enzymatic digestion (Digest-All Invitrogen; Thermo Fisher Scientific, Carlsbad, CA, USA) for $15 \mathrm{~min}$ at $37^{\circ} \mathrm{C}$ was used for pancytokeratin and FVIII; heat-induced antigen retrieval with microwave oven for $10 \mathrm{~min}$ at $567 \mathrm{~W}$ in citrate buffer $\mathrm{pH} 6.0$ for vimentin; heatinduced antigen retrieval with microwave oven for $10 \mathrm{~min}$ at $567 \mathrm{~W}$ in EDTA buffer $\mathrm{pH} 8.5$ for PNL2. No antigen retrieval was performed for S100. Blocking was obtained with a 1-h incubation at room temperature (RT) with horse normal serum for monoclonal and goat normal serum for polyclonal antibodies. Mouse monoclonal anti-pancytokeratin (clones AE1/AE3, Dako, Carpinteria, CA, USA; 1:2000 dilution), anti-vimentin (Clone 3B4, Dako; 1:1000 dilution) and anti-PNL2 (Clone PNL2, Monosan, Uden, The Netherlands; 1:25 dilution), and rabbit polyclonal anti-FVIII (Dako; 1:200 dilution) and anti-S100 (Dako; 1:500 dilution) antibodies were used. In negative controls, primary antibodies were replaced with normal rabbit or mouse serum (non-immune serum; Dako).

Primary antibodies were incubated overnight at $4^{\circ} \mathrm{C}$. Immunohistochemical reaction was visualized through an avidinbiotin complex method using biotinylated goat anti-rabbit (Vector Laboratories, Burlingame, CA, USA) and horse antimouse (Vector Laboratories) antibodies, and AEC (3-amino-9-ethylcarbazole) substrate-chromogen kit (Vector Laboratories). Sections were counterstain with Mayer's hematoxylin.

\section{Evaluation of melanin photo-degra- dation, cell morphology mainte- nance and immunohistochemical results}

To assess pigment photo-degradation and maintenance of cell morphology, sample sections subjected to different treatment duration have been compared each other and with control sections. Cell morphology was assessed on both neoplastic and all other cellular types present in sections (e.g., vessel endothelium, stromal cells, epithelial cell). For the evaluation of the immunohistochemical assay, the obtained results (positivity or negativity of the neoplastic cells for different markers) were compared with the expected results based on available literature.

\section{Results}

\section{Light source emission spectrum}

The emission spectrum of the light source (Figure 2) used to perform the treatments, was found to be distributed in the visible range of light radiation $(\sim 400-750$ $\mathrm{nm}$ ), with main peaks in the blue and greenyellow bands, around $460 \mathrm{~nm}$ and $540 \mathrm{~nm}$ respectively. In particular, it is important to remark that no emission was present in the UV wavelength range.

Temperatures reached by the sections during photobleaching treatment

The temperatures recorded inside the Petri dishes underwent a rapid increase, rising from $4^{\circ} \mathrm{C}$ before starting the treatment to $24^{\circ} \mathrm{C}$ after an hour from the beginning of the latter, remaining then stable at the same temperature for the following $11 \mathrm{~h}$.

\section{Melanin photobleaching and cell morphology maintenance}

Feline diffuse iris melanoma (Figure 3A). After a 24-h photobleaching treatment, neoplastic cells, and iris, ciliary and retinal pigment epithelial cells were completely bleached and no differences were noted for longer treatments (Figure 4). In all treated sections, morphology of neoplastic and non-neoplastic cells was maintained.

Canine dermal melanoma (Figure 3B). A 24-h treatment resulted in a considerable melanin degradation. Still, single or aggregated neoplastic melanocytes, either totally black or characterized by a black-brownish cytoplasm, were observed. Following a 2days treatment, neoplastic cells were entirely depigmented and no further changes were noted in the 3-, 4- and 5-days treatments. In all treated sections, morphology of neoplastic and non-neoplastic cells was maintained. Furthermore, the presence of an amelanotic area within the neoplasms allowed to observe that the morphology of amelanotic neoplastic cells did not change following treatments of different duration (Figure 5).

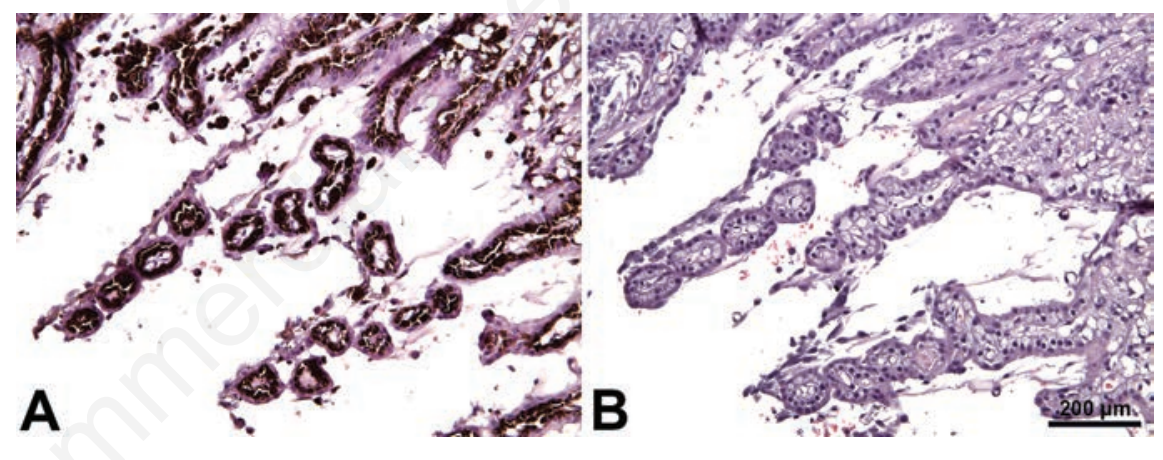

Figure 4. Ciliary bodies, pars plicata. Comparison of same field in a not-photobleached (A) and a 1-day photo-treated (B) section. Pigmented epithelium in specimen B was completely bleached and cell morphology was excellently maintained. H\&E staining.
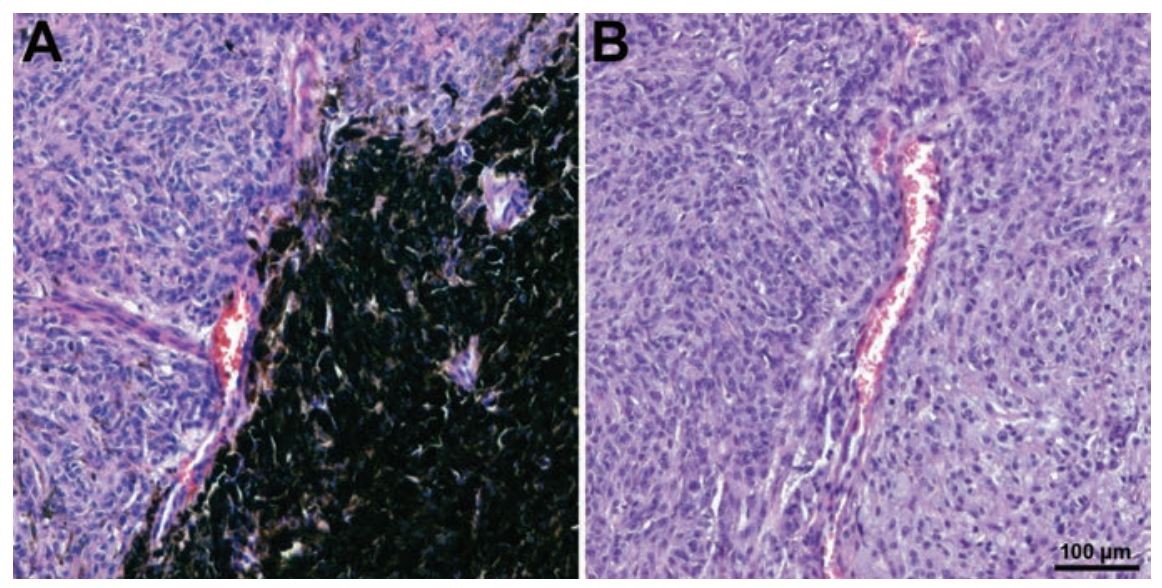

Figure 5. Canine dermal melanoma. Comparison of a not- (A) and a 5-day (B) phototreated section showing the border between an amelanotic and a melanotic area. Morphology of neoplastic cells is excellently maintained even after the treatment of maximum duration. H\&E staining. 
Gray horse dermal melanoma (Figure 3C). After a 24-h treatment, a considerable part of the section was still pigmented, with melanocytes characterized by a totally black cytoplasm or variable numbers of intracytoplasmic round black granules. After a 2-days treatment, occasional melanocytes with intracytoplasmic granules were still present. Melanin was not observed in the 3-, 4- and 5-days treated sections. In all treated sections, morphology of neoplastic and non-neoplastic cells was maintained.

Swine cutaneous melanoma (Figure 3D). In swine cutaneous pigmented melanoma, pigmented cells were completely bleached after a 24-h treatment. No changes were observed following longer treatments. In all treated sections, morphology of neoplastic and non-neoplastic cells was maintained.

\section{Immunohistochemical results}

In all tested sample neoplastic cells were widely positive for vimentin, S100 and PNL2, and negative for FVIII and pancytokeratin (Figure 6). Specifically, in all species, atypical cells showed a reticular cytoplasmic positivity for vimentin. Positivity for S100 ranged from diffuse (swine) to reticular-diffuse (all other species). PNL2 positive reaction had a granular cytoplasmic pattern, more pronounced in feline, canine and swine specimens than in equine sample. On the contrary, while positivity for FVIII and pancytokeratin was detected in the internal positive controls (Figure 7), melanocytic neoplastic cells resulted negative.

\section{Discussion}

This work aimed to asses if visible light, used to reduce background autofluorescence in fluorescence microscopy, can be effectively exploited in bright field microscopy to depigment FFPE melanocytic neoplasm sections. Indeed, light irradiation can permanently alter a molecule chemical structure causing the loss of its fluorescence. This phenomenon, named photobleaching, is usually feared because capable of reducing the signal of interest. It can be instead exploited in order to improve the ratio between signal of interest and nonspecific signal, damaging endogenous fluorescent molecules or reducing the adverse effects of histological procedures (e.g., autofluorescence induced by formalin fixation), prior to perform an immunofluorescence assay or a fluorescent staining. ${ }^{5-8}$ The usefulness of these protocols is also in the possibility to obtain a targeted effect by damag- ing fluorophores more seriously affected by irradiation of chosen wavelengths, compared to those that are not able to adsorb the same spectral range. ${ }^{5,6}$ In the present work, following the same principle, we evaluate whether it is possible to exploit melanin absorption spectra to determine its degradation in FFPE sections. To obtain a more targeted effect, we preferred to use an onlyvisible-light emitting light source to avoid an excessively deleterious effect on the samples deriving from the use of ultraviolet radiations. The latter, characterized by a higher energy content compared to visible radiations, are indeed absorbed also by nucleic acids, peptide groups and aromatic residues of proteins, most likely affecting the specificity of immune-histochemical and in situ hybridization results. ${ }^{9}$

A recent study demonstrated that a complete bleaching of human melanoma samples can be reached placing the sections in a $3 \% \mathrm{H}_{2} \mathrm{O}_{2}$ solution containing $1 \%$ formamide and irradiating with a $60 \mathrm{~W}$ incandescent light bulb. ${ }^{10}$ However, in this study, no control sections dipped in the same solution but not irradiated were used. Moreover, although the experiment was performed at RT, the temperature reached by the samples, due to both directly released heat and energy irradiated by the incandescent light bulb, has not been evaluated. It is in fact known that more than $80 \%$ of the energy radiated by incandescent light bulbs is represented by infrared radiations, invisible to the human eye but able to raise the temperature of the irradiated bodies. ${ }^{11}$ For these reasons and since a heated $3 \% \mathrm{H}_{2} \mathrm{O}_{2}$ solution can determine depigmentation of melanoma histological sections, it cannot be ruled out that the samples tested in that study benefited of a combined effect of $\mathrm{H}_{2} \mathrm{O}_{2}$ and heating. ${ }^{10} \mathrm{In}$ our study, particular attention was taken in maintaining previously-treated, intreatment and control sections in the same medium and at the same temperature, in order to avoid possible misleading effects deriving from variations of these parameters. Furthermore, the evaluation of the light source emission spectrum, allowed us to exclude a possible effect produced by infrared and ultraviolet radiations. For our purpose, four pigmented melanocytic neoplasms relatively common in veterinary histopathological routine diagnostic were chosen: a feline diffuse iris melanoma, a canine dermal melanoma, a gray horse dermal melanoma and a swine cutaneous melanoma. ${ }^{12-15}$ Our data demonstrated that a complete melanin photo-degradation can be achieved using a visible-light emitting light source, even if different samples required different light exposure times. Feline and swine sections were completely bleached following a 1-day treatment, while 2 and 3 days were required to obtain the same result in the canine and equine samples, respectively. The long exposure time required by the equine sample to reach complete depigmentation can be explained in light of coat color and melanoma pathogenesis in gray horses. In these horses, dermal melanoma development is linked to specific duplications of genomic regions containing binding sites for proteins involved in melanocyte gene expression and metabolism. This genomic modification brings to eumelanin overproduction and to an increased proliferative boost. ${ }^{14}$ It can be therefore hypothesized that the predominant pigment in the equine sample was eumelanin, less prone to photo-degradation than pheomelanin. ${ }^{3,16}$ Moreover, since the same genetic alteration leads to eumelanin overproduction, the time required to bleach numerous melanin molecules is longer than the one required for fewer molecules. ${ }^{14}$

In feline iris melanoma, neoplastic cell bleaching was achieved with a 1-day light exposure. Although a variation in melanin content between feline and equine neoplastic cells cannot be ruled out, it can be also hypothesized that the difference in the photobleaching time can be explained by a different eu- and pheomelanin content. Specifically, while gray horse dermal melanoma contains eumelanin, pheomelanin, that is known to be more prone to photo-degradation than eumelanin, is the melanin suspected to be predominant in feline iris melanocytes. . $^{3,12,14,16}$ It is interesting to note that the same irradiation period was able to completely bleach not only neoplastic pigmented cells but also iris, ciliary bodies and retinal pigmented epithelial cells, derived from the embryonic optical cup of neural ectoderm, producing neuromelanin granules, composed of both euand pheomelanin. ${ }^{2}$ It can be hypothesized that this phenomenon was due to the treatment of $24 \mathrm{~h}$. Shorter treatments could perhaps show more subtle difference in depigmentation times of the various pigmented cell types present in the sample. Obviously, also in this last case it is not possible to exclude that the present results are due to a different proportion of eu/pheo-melanin content in the pigmented cell populations present in the section. Lack of studies concerning melanin contents and types in canine and swine cutaneous melanomas makes it difficult to interpret the results obtained in these species. Despite this, it is important to underline that in all samples, the pigment was completely removed, albeit with different exposure times.

The consistency between the available literature and the results obtained combining photobleaching with immunohistochemistry shows that photobleaching does 


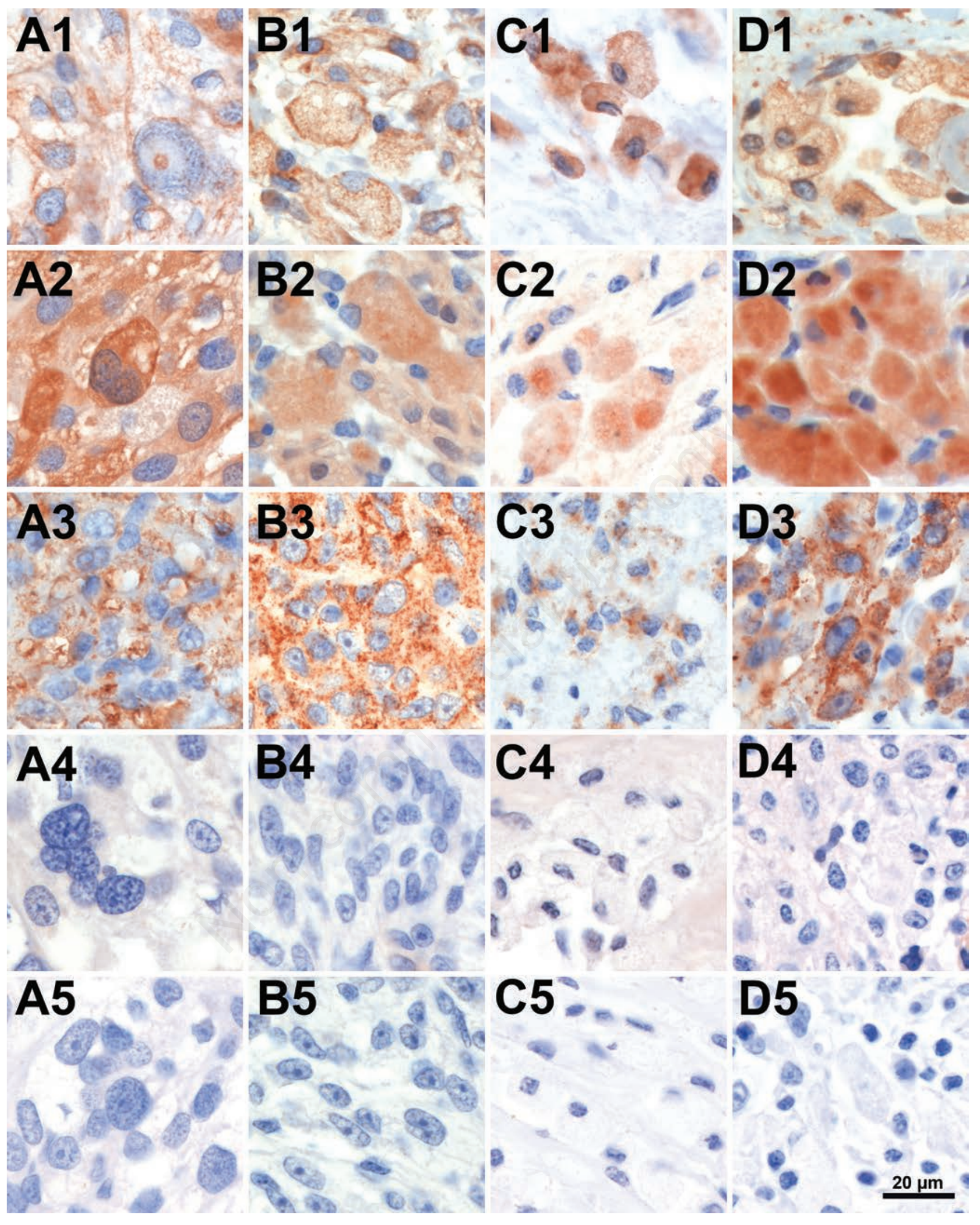

Figure 6. Results of the combined photobleaching-immunohistochemical protocol on the feline (A), the canine (B), the equine (C) and the swine (D) specimens. In all tested samples neoplastic cells resulted positive for vimentin (1), S100 (2) and PNL2 (3), and negative for FVIII (4) and pancytokeratin (5). Immunohistochemistry, Mayer's hematoxylin counterstain. 
not affect immunoreactivity of tested markers within FFPE samples. ${ }^{12,15,17-21}$ The potential of this new bleaching method is that, compared to traditional chemical bleaching methods, photo-induced bleaching should offer a greater oxidative selectivity, due to the use of light at wavelengths preferentially absorbed by the target molecule, with a hypothetical greater preservation of investigable molecules. ${ }^{5,6,23-25}$

The chemical mechanisms involved in melanin degradation acts in a sort of autolimiting fashion: the oxidation induced by the reactive oxygen species, produced during melanin photo-irradiation, degrades melanin itself. Consequently, the variation of the absorption spectrum derived by melanin degradation would no longer allow the formation of reactive oxidizing species. ${ }^{3,4}$ On the contrary, the oxidizing effect of solutions routinely used to bleach FFPE sections, being absent the targeted effect ensured by melanin absorption spectrum and light source emission spectrum, would continue even after the complete melanin degradation. Furthermore, it can be hypothesized that a selective effect can also be achieved with molecules other than proteins, such as nucleic acids that could be damaged by $\mathrm{H}_{2} \mathrm{O}_{2}$, a chemical oxidant routinely used to bleach histological sections. ${ }^{9,26-28}$ Although melanin photo-degradation involves the production of reactive oxygen species, the rapid quench of these oxidizing species by melanin itself should guarantee a lower oxidizing effect on nucleic acids, compared with the routinely used bleaching solutions. ${ }^{3,4}$ Thus, photo-induced bleaching could have a potential use working with in situ hybridization techniques.

In the present study, during photobleaching, sections were placed in TRIS buffer $\mathrm{pH} 7.6$ added with $0.5 \%$ sodium azide to prevent bacterial growth. Sodium azide can have a dual behavior in photooxidation events, acting either as a strong singlet oxygen quencher or as an azide radical or hydroperoxide producer. In particular it would behave as a pro-oxidant in acidic environments and as an anti-oxidant in neutral environments. ${ }^{29,30}$ Since TRIS buffer was the dipping medium in the present experiment, it cannot be ruled out that sodium azide has slowed down the photobleaching process quenching the reactive oxygen species produced during melanin photo-degradation process. On the contrary, a possible pro-oxidant effect of sodium azide can be excluded since the dipping solution was not an acidic medium.

In conclusion, this pilot study demonstrates that visible light can be effectively exploited to completely bleach pigmented melanoma FFPE sections, together with an excellent maintenance of cell morphology. However, a study involving a larger number of samples is necessary to certainty confirm what has been observed in this preliminary experiment. Even though this protocol is still not practical for diagnostic routine, due to time and special equipment requirements, the obtained results open interesting perspective on the study of melanin in FFPE samples. In order to improve the feasibility of the described protocol, one of the first steps to follow would be to reduce the time needed to achieve complete section photobleaching. This achievement could be reached by exploiting different variables such as incubation temperature, spectral selection and energy radiated by incident light, and the use of a sodium azide-free dipping solution.

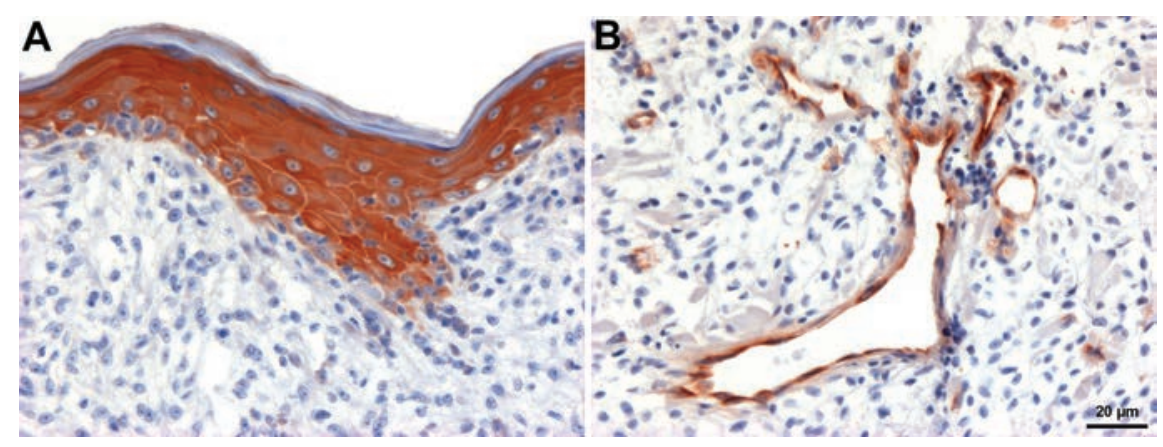

Figure 7. Canine dermal melanoma. The epidermis stained intensely pancytokeratin positive, neoplastic cells are diffusely negative (A). Blood vessel endothelium was FVIII positive, neoplastic cells were negative (B). Immunohistochemistry, Mayer's hematoxylin counterstain.

\section{References}

1. Solano F. Melanins: Skin pigments and much more-Types, structural models, biological functions, and formation routes. New J Sci 2014;2014. Doi: 10.1155/2014/498276.

2. Bush WD, Garguilo J, Zucca FA, Albertini A, Zecca L, Edwards GS, et al. The surface oxidation potential of human neuromelanin reveals a spherical architecture with a pheomelanin core and a eumelanin surface. Proc Natl Acad Sci USA 2006;103:14785-9.

3. Ito $\mathrm{S}$, Wakamatsu $\mathrm{K}$, Sarna $\mathrm{T}$. Photodegradation of eumelanin and pheomelanin and its pathophysiological implications. Photochem Photobiol 2018;94: 409-20. Doi: 10.1111/ php. 12837.

4. Szewczyk G, Zadlo A, Sarna M, Ito S, Wakamatsu K, Sarna T. Aerobic photoreactivity of synthetic eumelanins and pheomelanins: generation of singlet oxygen and superoxide anion. Pigment Cell Melanoma Res 2016;29:669-78. Doi: 10.1111/pcmr.12514.

5. Neumann M, Gabel D. Simple method for reduction of autofluorescence in fluorescence microscopy. J Histochem Cytochem 2002;50:437-9. Doi: 10.1177/002215540205000315.

6. Duong H, Han M. A multispectral LED array for the reduction of background autofluorescence in brain tissue. J Neurosci Methods 2013;220:46-54. Doi: 10.1016/j.jneumeth.2013.08.018.

7. Kumar BS, Sandhyamani S, Nazeer SS, Jayasree RS. Rapid and simple method of photobleaching to reduce background autofluorescence in lung tissue sections. Indian J Biochem Biophys 2015;52:107-10.

8. Sun Y, Ip P, Chakrabartty A. simple elimination of background fluorescence in formalin-fixed human brain tissue for immunofluorescence microscopy. J Vis Exp 2017;:e56188. Doi: 10.3791/56188.

9. Schmid FX. Biological macromolecules: UV-visible spectrophotometry. In S. Robinson, E. Ayres, Editors, Encyclopedia of Life Sciences; J. Wiley \& Sons, Hoboken; 2001. Doi: 10.1038 /npg.els.0003142.

10. Jordan T, Williams D, Criswell S, Wang Y. Comparison of bleaching protocols utilizing hematoxylin and eosin stain and immunohistochemical proliferation marker MCM3 in pigmented melanomas. J Histotechnol 2019;16:16. Doi: 10.1080/01478885. 2019. 1649886.

11. Elvidge CD, Keith DM, Tuttle BT, Baugh KE. Spectral identification of 
lighting type and character. Sensors 2010;10:3961-88. Doi: 10.3390/s100 403961

12. Wiggans KT, Reilly CM, Kass PH, Maggs DJ. Histologic and immunohistochemical predictors of clinical behavior for feline diffuse iris melanoma. Vet Ophthalmol 2016;19(S1):44-55. Doi: 10.1111/vop. 12344 .

13. Nishiya AT, Massoco CO, Felizzola CR, Perlmann E, Batschinski K, Tedardi MV, et al. Comparative aspects of canine melanoma. Vet Sci 2016;3. pii: E7. Doi: 10.3390/vetsci3010007.

14. Teixeira RB, Rendahl AK, Anderson SM, Mickelson JR, Sigler D, Buchanan $\mathrm{BR}$, et al. Coat color genotypes and risk and severity of melanoma in gray quarter horses. J Vet Intern Med 2013;27:1201-8. Doi: 10.1111/ jvim. 12133 .

15. Grossi AB, Hyttel P, Jensen HE, Leifsson PS. Porcine melanotic cutaneous lesions and lymph nodes: immunohistochemical differentiation of melanocytes and melanophages. Vet Pathol 2015;52:83-91. Doi: 10.1177/0300985814521637.

16. Swope VB, Abdel-Malek ZA. MC1R: Front and center in the bright side of dark eumelanin and DNA repair. Int J Mol Sci 2018;19. pii: E2667. Doi: 10.3390/ijms19092667.

17. Grahn BH, Peiffer RL, Cullen CL, Haines DM. Classification of feline intraocular neoplasms based on morphology, histochemical staining, and immunohistochemical labelling. Vet Ophthalmol 2006;9:395-403. Doi:10. 1111/j.1463-5224.2006. 00479.x.
18. Smedley RC, Lamoureux J, Sledge DG, Kiupel M. Immunohistochemical diagnosis of canine oral amelanotic melanocytic neoplasms. Vet Pathol 2011;48:32-40. Doi: 10.1177/030098 5810387447.

19. Nordio L, Fattori S, Vascellari M, Giudice C. Evidence of vasculogenic mimicry in a palpebral melanocytoma in a dog. J Comp Pathol 2018;162:4346. Doi: 10.1016/j.jcpa.2018.06.003.

20. Ramos-Vara JA, Frank CB, DuSold D, Miller MA. Immunohistochemical expression of melanocytic antigen PNL2, Melan A, S100, and PGP 9.5 in equine melanocytic neoplasms. Vet Pathol 2014;51:161-6. Doi: 10.1177/0300985812471545.

21. Bottegaro NB, Gotic J, Babic NP, Zagradisnik LM, Kurilj AG. Abrupt exacerbation of a mid-tail melanoma during late pregnancy in a bay Holsteiner mare. Wien Tierärzt Monat 2018;105:25-31.

22. Perez J, García PM, Bautista MJ, Millán Y, Ordás J, Martín de las Mulas J. Immunohistochemical characterization of tumor cells and inflammatory infiltrate associated with cutaneous melanocytic tumors of Duroc and Iberian swine. Vet Pathol 2002;39:44551. Doi: 10.1354/vp.39-4-445.

23. Shen H, Wu W. Study of melanin bleaching after immunohistochemistry of melanin-containing tissues. Appl Immunohistochem Mol Morphol 2015;23:303-7. Doi: 10.1097 /PAI.0000000000000075.

24. Foss AJ, Alexander RA, Jefferies LW, Lightman S. Immunohistochemical tech- niques: the effect of melanin bleaching. Br J Biomed Sci 1995;52:22-5.

25. Orchard GE, Calonje E. The effect of melanin bleaching on immunohistochemical staining in heavily pigmented melanocytic neoplasms. Am J Dermatopathol 1998;20:357-61. Doi: 10.1097/00000372-199808000-00006.

26. Driessens N, Versteyhe S, Ghaddhab C, Burniat A, De Deken X, Van Sande J, et al. Hydrogen peroxide induces DNA single- and double-strand breaks in thyroid cells and is therefore a potential mutagen for this organ. Endocr Relat Cancer 2009;16:845-56. Doi: 10.1677/ ERC-09-0020.

27. Olson MJ. DNA strand breaks induced by hydrogen peroxide in isolated rat hepatocytes. J Toxicol Environ Health 1988;23:407-23. Doi: 10.1080/152873 98809531123

28. McDonald RJ, Pan LC, St George JA, Hyde DM, Ducore JM. Hydrogen peroxide induces DNA single strand breaks in respiratory epithelial cells. Inflammation 1993;17:715-22. Doi: 10.1007/BF00920476.

29. An S, Lee E, Choe E. Effects of solubility characteristics of sensitiser and $\mathrm{pH}$ on the photooxidation of oil in tuna oiladded acidic $\mathrm{O} / \mathrm{W}$ emulsions. Food Chem 2011;128:358-63. Doi: 10.1016/j.foodchem.2011.03.034.

30. Bancirova M. Sodium azide as a specific quencher of singlet oxygen during chemiluminescent detection by luminol and Cypridina luciferin analogues. Luminescence 2011;26:685-8. Doi: 10.1002/bio. 1296 . 